\title{
Cambios en la etiología, resultados y características de los pacientes con hemorragia digestiva aguda grave a lo largo del periodo 1999-2005
}

\author{
A. Garrido, J. L. Márquez, F. J. Guerreroํㅗ E. Leo, M. A. Pizarro y C. Trigo \\ Unidad de Sangrantes. Hospital Virgen del Rocío. Sevilla. ${ }^{~}$ Servicio de Medicina Interna. Hospital Virgen Macarena. \\ Sevilla
}

\section{RESUMEN}

Objetivos: analizar la evolución, a lo largo del periodo 19992005, de las siguientes variables de los pacientes ingresados por hemorragia digestiva (HD) en una Unidad de Sangrantes: etiología, patología de base, consumo de AINE/anticoagulación y mortalidad.

Material y métodos: durante el periodo 1999-2005 se ha estudiado la evolución de las siguientes causas de HD que requirieron ingreso en la Unidad de Sangrantes: ulcus duodenal (UD), ulcus gástrico (UG), hipertensión portal (HPT) y otros. De igual forma se ha analizado la evolución en el porcentaje de enfermos ingresados con patología de base, consumo de fármacos AINE/anticoagulantes y mortalidad.

Resultados: se han incluido 1.611 pacientes en el estudio con una edad media de 60,45 años (59,7-61,2), 76,41\% hombres $(74,3-78,5)$. La UD fue responsable del $22,20 \%$ de episodios $(20,2-24,3)$, la UG del $18,40 \%(16,6-20,4)$ y la HPT del $33,60 \%$ $(31,3-36,0)$.

De forma global realizaban tratamiento con AINE el 34,5\% (32,6-37,3), anticoagulación el 7,1\% $(6,0-8,6)$, presentaban patología de base el $72,6 \%(70,4-74,8)$ y la mortalidad global fue del $6,27 \%(5,16-7,59)$. A lo largo del periodo estudiado se constató un aumento de los pacientes con patología de base $(p<0,02)$ y un descenso en los ingresos por UD ( $p<0,04)$, permaneciendo estables el resto de variables estudiadas.

Conclusiones: la UD y UG junto con la HPT suponen el 75\% de los ingresos en la Unidad de Sangrantes. A lo largo de los últimos 7 años se constata un descenso de los casos debido a UD y un aumento de pacientes con patología de base, mientras la mortalidad global se mantiene estable.

Palabras clave: Etiología. Epidemiología. Hemorragia digestiva. Úlcera gastroduodenal. Hemorragia varicosa.

\begin{abstract}
Objectives: to analyze the evolution of the following variables in patients admitted to a Blood Unit for gastrointestinal bleeding throughout 1999-2005: etiology, comorbid diseases, use of NSAIDs/anticoagulants, and mortality.

Material and methods: we analyzed the evolution of the following causes of GIB that required admission to the Blood Unit from 1999 to 2005: duodenal ulcer (DU), gastric ulcer (GU), portal hypertension (PHT), and others. We also analyzed changes in the percentage of patients admitted with comorbid disease, use of NSAIDs/anticoagulants, and mortality.

Results: 1,611 patients with a mean age of 60.45 years (59.7-61.2) were included in this study; $76.41 \%$ were males (74.3-78.5). DU was the cause of bleeding in $22.20 \%$ of cases (20.2-24.3), GU in $18.40 \%$ of cases (16.6-20.4), and PHT in $33.60 \%$ of cases (31.3-36.0).

In all, $34.5 \%(32.6-37.3)$ of patients were taking NSAIDs, $7.1 \%(6.0-8.6)$ were receiving anticoagulant therapy, $72.6 \%$ (70.4-74.8) presented with comorbid disease, and overall mortality was $6.27 \%$ (5.16-7.59). Throughout the $1999-2005$ period there was an increase in the number of patients with comorbid diseases $(p<0.02)$, and a decrease in cases of DU $(p<0.04)$, without significant differences in the remaining variables.

Conclusions: DU, GU and PHT account for three quarters of admissions to our Blood Unit. Over the last seven years, there has been a decrease in cases due to DU, and an increase in patients with comorbid disease; overall mortality rates have remained stable.
\end{abstract}

Key words: Etiology. Epidemiology. Gastrointestinal bleeding. Gastroduodenal ulcer. Variceal bleeding.

Garrido A, Márquez JL, Guerrero FJ, Leo E, Pizarro MA y Trigo C. Cambios en la etiología, resultados y características de los pacientes con hemorragia digestiva aguda grave a lo largo del periodo 1999-2005. Rev Esp Enferm Dig 2007; 99: $275-279$.

Recibido: 28-09-06.

Aceptado: 25-01-07.

Correspondencia: Antonio Garrido Serrano. Servicio de Aparato Digestivo. Hospital General Universitario Virgen del Rocío. Avda. Manuel Siurot, s/n. 41013 Sevilla. e-mail: agarser@telefonica.net

\section{INTRODUCCIÓN}

La hemorragia digestiva (HD) aguda constituye hoy en día un problema médico que conlleva una significativa morbilidad, mortalidad y un elevado consumo de recur- 
sos sanitarios (1); tradicionalmente se ha dividido según su lugar de origen en alta y baja (proximal o distal al ángulo de Treitz), y según su etiología como aquella HD de origen varicoso (HPT) y no varicoso (cuyo principal responsable es la úlcera gastroduodenal), cuya metodología diagnóstica, tratamiento y pronóstico son diferentes (2).

Debido al progreso de la medicina en general y de la gastroenterología en particular, se podría esperar un descenso en la incidencia y un mejor pronóstico en pacientes con HD en los últimos años, pero por idéntico motivo cada vez ingresan pacientes de edad más avanzada y pluripatológicos por lo que tales parámetros no se han modificado sustancialmente (3). La erradicación de Helicobacter pylori, la alta incidencia de hepatitis virales y elevado consumo de alcohol está aumentando el porcentaje de hemorragias varicosas frente a las originadas por úlcera gastroduodenal (4).

El análisis de las tendencias en cuanto a etiología y factores de riesgo asociados a un peor pronóstico en pacientes con HD a lo largo de los últimos años, permitirá la implantación de medidas de profilaxis específicas enfocadas a conseguir disminuir la incidencia y mejorar los resultados en estos enfermos (5).

En este trabajo nos proponemos evaluar la evolución en cuanto a etiología, características de los pacientes y resultados de los enfermos ingresados por HD aguda en la Unidad de Sangrantes de un hospital de tercer nivel.

\section{MATERIAL Y MÉTODOS}

Se ha realizado un estudio que abarca el periodo comprendido entre 1999 y 2005, de todos los enfermos ingresados tanto por hemorragia digestiva alta como baja en la Unidad de Sangrantes del Hospital Virgen del Rocío de Sevilla (hospital de tercer nivel, que atiende a una población de 735.973 habitantes), que son aquellos pacientes sangrantes que presentan inestabilidad hemodinámica o cuyas características hagan prever la posibilidad de persistencia o recidiva hemorrágica.

En todos los casos se recogieron datos de filiación como sexo y edad en el momento del ingreso; antecedentes personales de hábito tabáquico y enólico, entendiendo el primero como el consumo de más de 10 cigarrillos/día y el segundo como el consumo regular de más de $80 \mathrm{~g}$ de etanol/día en varones y 40 en mujeres. El antecedente del consumo de aspirina u otros AINE, así como de anticoagulantes orales se consideró cuando quedó reflejado de forma inequívoca en la historia clínica por parte del paciente o sus familiares.

Se evaluó la presencia o no de enfermedades concomitantes de cualquier órgano o sistema vital (cardiovascular, pulmonar, renal, etc.).

En cuanto al episodio hemorrágico se clasificó como: hemorragia digestiva alta (originada entre el esfínter esofágico superior y el ángulo de Treitz) o baja (desde ángulo de Treitz hasta margen anal). También se evaluó la forma de presentación clínica de la hemorragia bien como hemate- mesis, melenas, hematoquecia o rectorragia, así como la situación hemodinámica del paciente definida como estable cuando se cumplían los siguientes criterios: presión arterial sistólica superior a $100 \mathrm{mmHg}$, frecuencia cardiaca inferior a 100 latidos/minuto, ausencia de cambios en la presión arterial y frecuencia cardiaca tras el ortostatismo, ausencia de signos clínicos de hipoperfusión periférica y diuresis superior a $30 \mathrm{ml} / \mathrm{h}$; e inestabilidad hemodinámica cuando se cumplían dos o más de los siguientes criterios: presión arterial sistólica inferior a $100 \mathrm{mmHg}$, frecuencia cardiaca superior a 100 sístoles/minuto, signos clínicos de hiperfusión periférica y cambios significativos en la presión arterial y frecuencia cardiaca tras el ortostatismo.

Se anotó la etiología de la hemorragia digestiva alta clasificándola como sigue: UD, UG, Mallory-Weiss, HPT, hemorragia alta de origen oscuro (HDAO) y otros; la hemorragia digestiva baja se clasificó según su etiología en: enfermedad diverticular, angiodisplasias, hemorragia digestiva baja de origen oscuro (HDBO) y otros.

La evolución del paciente se clasificó en: a) favorable: cuando no existió evidencia de nuevos episodios de exteriorización hemorrágica, la situación hemodinámica se mantuvo estable y no hubo descensos del hematocrito; b) recidiva hemorrágica: en las primeras 48 horas si la necesidad de perfusión de fluidos era superior a $1.000 \mathrm{ml} / \mathrm{h}$ para mantener la estabilidad hemodinámica, o bien se producía un descenso de al menos 7 puntos de hematocrito acompañado de "melenes frescas" o nuevos episodios de hematemesis o rectorragias. A partir del tercer día se consideró recidiva cuando se presentaron nuevos episodios de exteriorización hemorrágica; y c) exitus del paciente.

\section{Estudio estadístico}

Se incluyeron diversas variables cuantitativas y cualitativas, expresándolas como media o porcentaje respectivamente, con intervalos de confianza del 95\%. Se realizó test de ANOVA 1 factor para comparar la evolución de las diferentes variables a lo largo de los años estudiados. La significación estadística se declaró para una $\mathrm{p}<0,05$. El análisis estadístico se realizó con el programa SPSS 12.

\section{RESULTADOS}

Durante el periodo 1999-2005 se han incluido en este estudio 1.611 pacientes que han ingresado en la Unidad de Sangrantes del Servicio de Aparato Digestivo del Hospital Virgen del Rocío de Sevilla, 76,41\% hombres (74,3$78,5)$ y $23,59 \%$ mujeres $(21,4-25,8)$ con una edad media de 60,45 $\pm 15,59$ años $(59,7-61,2)$.

En la tabla I se muestran las características generales del total de pacientes de la muestra en cuanto a sus antecedentes, forma de presentación y diagnóstico etiológico; a destacar que el 36,70\% referían antecedentes de hábito etílico y el 34,5\% tratamiento previo con AINE, la úlcera 
Tabla I. Características generales de la muestra $(n=1.611)$

\begin{tabular}{lcc}
\hline Variables & Media & IC $95 \%$ \\
\hline A. Datos generales & & \\
\hline Edad (años) & 60,45 & $59,7-61,2$ \\
Hombres (\%) & 76,41 & $74,3-78,5$ \\
Hábito tabáquico (\%) & 26,70 & $24,6-28,9$ \\
Hábito enólico (\%) & 36,70 & $34,3-39,1$ \\
Tto. previo con AlNE (\%) & 34,50 & $32,6-37,3$ \\
Tto. con anticoagulantes (\%) & 7,10 & $6,0-8,6$ \\
\hline B. Presentación clínica de la hemorragia & & \\
\hline Hematemesis (\%) & 50,22 & $47,8-52,7$ \\
Melenas (\%) & 53,88 & $51,4-56,3$ \\
Rectorragia (\%) & 13,70 & $12,0-15,5$ \\
Hematoquecia (\%) & 2,60 & $1,9-3,5$ \\
\hline C. Diagnóstico etiológico & & \\
\hline Úlcera gástrica (\%) & \multicolumn{3}{c}{} \\
Úlcera duodenal (\%) & 18,40 & $16,6-20,4$ \\
Mallory-Weiss (\%) & 22,20 & $20,2-24,3$ \\
Angiodisplasia (\%) & 5,00 & $4,0-6,30$ \\
Hipertensión portal (\%) & 1,60 & $1,0-2,30$ \\
Diverticulosis (\%) & 33,60 & $31,3-36,0$ \\
HDA de origen oscuro (\%) & 3,60 & $2,8-4,70$ \\
HDB de origen oscuro (\%) & 1,70 & $1,1-2,20$ \\
Otros (\%) & 3,30 & $2,5-4,30$ \\
Tto. & 10,70 & $9,4-12,5$
\end{tabular}

Tto.: tratamiento. AINE: antinflamatorios no esteroideos; HDA: hemorragia digestiva alta; HDB: hemorragia digestiva baja.

gastroduodenal fue la responsable del $40,60 \%$ de los ingresos $(18,40 \%$ UG y $22,20 \%$ UD) y la hemorragia por HPT en el 33,60\% de los casos, es decir, sumadas ambas etiologías alcanzan las tres cuartas partes del total de los ingresos en la Unidad de Sangrantes.

La tabla II muestra que el 59,4\% de los pacientes a su ingreso presentaban inestabilidad hemodinámica y el $72,63 \%$ patología de base, con una mortalidad global del $6,27 \%$, significativamente superior en los casos de hemorragia varicosa frente a los debidos a úlcera gastroduoenal $(\mathrm{p}<0,01)$.

Empleando el test de ANOVA se constató una menor incidencia de hemorragia por UD a lo largo del periodo estudiado, así como cada vez mayor porcentaje de pacientes con patología de base (Figs. 1 y 2 ) y proporción de hemorragias de origen oscuro, tanto altas como bajas (Fig. 3); no se encontraron diferencias en cambio a

Tabla II. Características generales de la muestra $(n=1.611)$

\begin{tabular}{lccc}
\hline Variables & Media & IC 95\% & $p$ \\
\hline Inestabilidad hemodinámica (\%) & 59,40 & $56,96-61,81$ & \\
Patología de base (\%) & 72,63 & $70,36-74,78$ & \\
Mortalidad global (\%) & 6,27 & $5,16-7,59$ & \\
Mortalidad por HPT (\%) & 10,43 & $8,91-12,02$ & \\
Mortalidad por UD (\%) & 3,04 & $2,51-3,73$ & $<0,01$ \\
Mortalidad por UG (\%) & 3,86 & $3,15-4,61$ &
\end{tabular}

HPT: hemorragia por hipertensión portal; UD: hemorragia por ulcus duodenal; UG: hemorragia por ulcus gástrico.

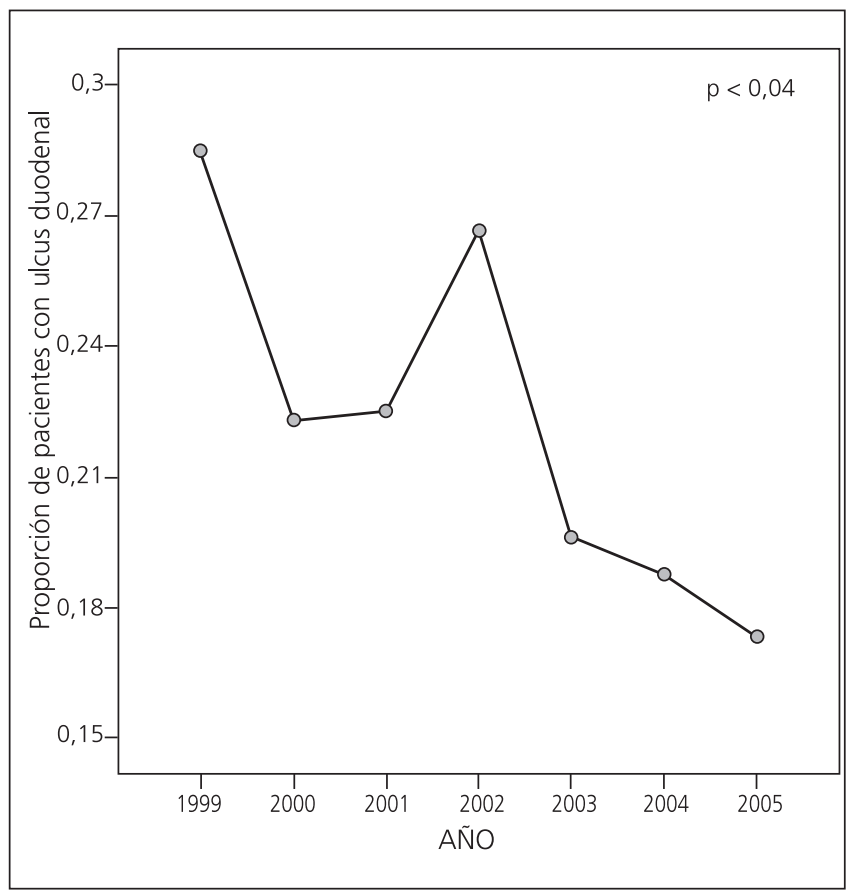

Fig. 1. Evolución de los ingresos por úlcera duodenal durante los años 1999-2005.

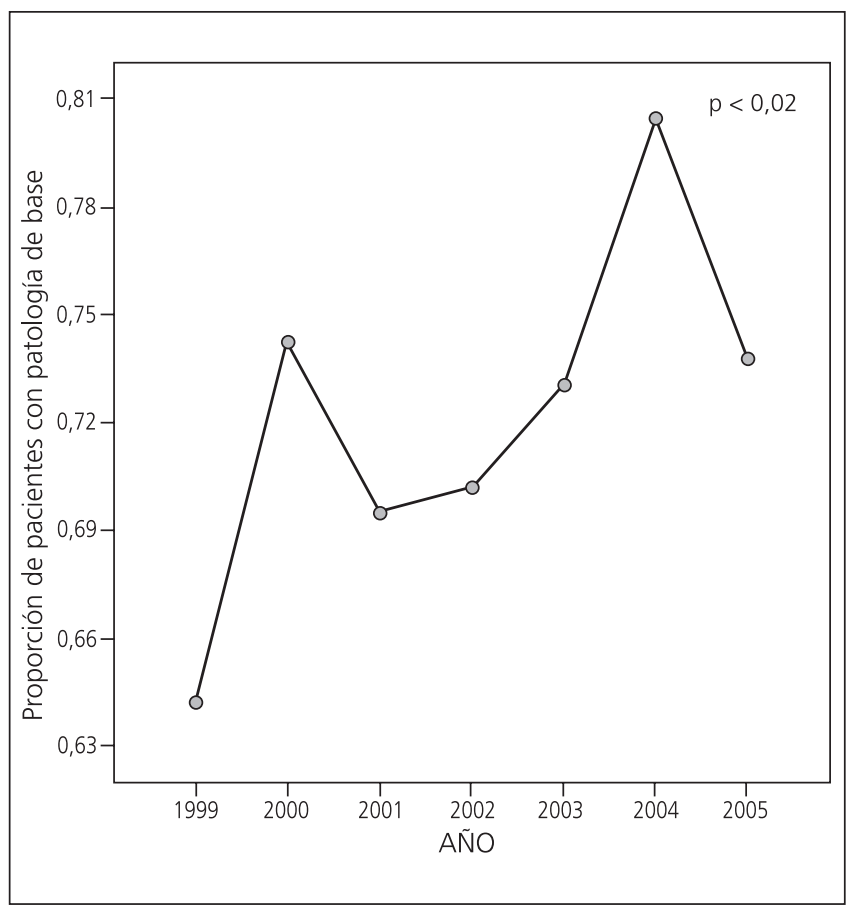

Fig. 2. Evolución de los ingresos de pacientes con patología de base durante el periodo 1999-2005.

lo largo de los años del estudio en la incidencia de hemorragia por UG ni por HPT ( $\mathrm{p}=\mathrm{NS})$.

Tampoco se encontraron diferencias a lo largo del periodo estudiado en cuanto a forma de presentación clínica, presencia de inestabilidad hemodinámica, tratamiento con AINE o anticoagulantes y mortalidad. 


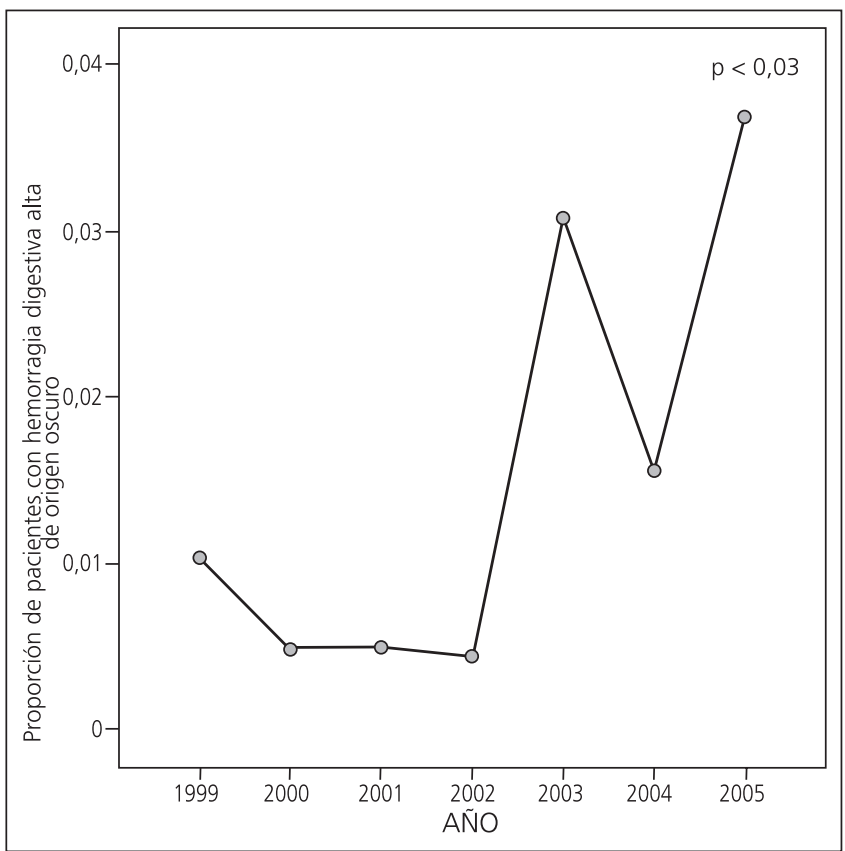

Fig. 3. Evolución de los ingresos por hemorragia digestiva alta oscura en el periodo estudiado.

\section{DISCUSIÓN}

La hemorragia digestiva alta tiene una incidencia anual de 50-150 casos por cada 100.000 habitantes (6), siendo la úlcera péptica gastroduodenal la responsable del $60-70 \%$ de los casos, seguida de la hemorragia por varices esófago-gástricas $(1,6)$.

En nuestra Unidad de Sangrantes las hemorragias por UD suponen el $22,20 \%$ de los ingresos y las debidas a UG el 18,40\%, por lo que la úlcera péptica es responsable del $40,6 \%$ del total de ingresos, cifra sensiblemente inferior a la que se comunicaba hace algunos años, y ello es debido a una incidencia significativamente cada vez menor de HD por UD.

A finales de la década de los 80 y principios de los 90 aún no se había constatado una disminución en el número de admisiones hospitalarias como consecuencia de la úlcera sangrante $(2,7)$, pero en los últimos 10 años y como consecuencia del cada vez mayor volumen de prescripción de fármacos inhibidores de la bomba de protones (unido a su menor coste), así como de la erradicación de Helicobacter pylori que previene la recidiva ulcerosa y sus complicaciones, se está asistiendo a una disminución de la incidencia de la úlcera péptica como causa de hemorragia digestiva (8); así por ejemplo, en una reciente serie de 7.822 pacientes con hemorragia digestiva alta, la úlcera péptica sólo supuso el 20,6\% de los casos (9).

Las dos causas más importantes de úlcera péptica y sus complicaciones son la infección por Helicobacter pylori y la ingesta de AINE (10), el tratamiento y posterior erradicación de la infección en pacientes jóvenes está modifi- cando el perfil del paciente con ulcus activo y sus complicaciones (11), a favor del paciente consumidor de aspirina/AINE/anticoagulado, que por lo general suelen ser personas de edad avanzada y con patología de base concomitante (12).

Así en nuestra serie el 34,5\% de pacientes referían ingesta de AINE y el 7,1\% tratamiento anticoagulante, y además hemos constatado a lo largo de los años un incremento de los pacientes con patología de base, que por lo general se trata de personas de edad avanzada, frecuentemente con varias enfermedades asociadas y polimedicados. Este hecho explicaría, al menos en parte, que la tasa de mortalidad en la hemorragia por ulcus no haya experimentado un descenso significativo en las últimas décadas, a pesar de los espectaculares avances clínicos, endoscópicos y farmacológicos (3).

En cambio la mortalidad por hemorragia aguda causada por varices esofágicas ha disminuido considerablemente en las últimas dos décadas, desde aproximadamente un $40 \%$ en los años ochenta (13) hasta menos de un $20 \%$ en la actualidad (14); este descenso se debe por un lado a una mejora en el manejo agudo de la hemorragia, así como a una mejor comprensión de la historia natural y de la fisiopatología de la formación, progresión y rotura de las varices, que han permitido aplicar tratamientos farmacológicos, endoscópicos y radiológicos intervencionistas de alta eficacia (15). Así en la actualidad el hepatocarcinoma ha relegado al segundo lugar a la HD como causa de muerte en el paciente cirrótico (16), no obstante aunque la mortalidad ha descendido, no ha ocurrido igual con su incidencia en los últimos años, que se ha mantenido estable o incluso en aumento, siendo responsable de una elevada morbilidad, así como de un importante consumo de recursos sanitarios.

En un reciente estudio multicéntrico epidemiológico realizado en Francia durante el periodo 1996-2000, la hemorragia varicosa fue tan frecuente como la debida a ulcus péptico y se constató una disminución significativa de su mortalidad que fue del 11,1\% en el año 2000 (17); en nuestra serie la hemorragia por HPT del cirrótico ha supuesto la tercera parte del total de los ingresos en la Unidad, con una mortalidad media del $10,43 \%$ que se ha mantenido estable a lo largo del periodo estudiado.

A diferencia del UD donde la erradicación de Helicobacter pylori ha hecho disminuir su incidencia y complicaciones, la ingesta de alcohol, que la Organización Mundial de la Salud sitúa como tercera causa de discapacidad en el mundo occidental (18) y frecuente causa de hospitalización (19), así como la epidemia de infección crónica por el virus de la hepatitis C, iniciada en los años 60 y que alcanzó su pico a mediados de los 80 , cuyas consecuencias estamos viendo ahora y seguiremos viendo al menos en las próximas dos décadas (20), hacen que en los años venideros no se prevea un descenso en la incidencia de hemorragia varicosa.

En la mayoría de los pacientes con hemorragia digestiva, el origen del sangrado se identifica con las pruebas 
diagnósticas convencionales, sin embargo en el $5 \%$ de los casos, igual que en nuestra serie, persiste el sangrado sin llegarse a un diagnóstico mediante estos métodos, es lo que se denomina hemorragia de origen oscuro (HDOO) (21).

Si bien algún estudio aislado no encuentra diferencias en cuanto a número de días de hospitalización, requerimientos transfusionales, necesidad de cirugía y pronóstico de estos pacientes frente a otras causas de hemorragia (22), la mayoría de autores coinciden en que el manejo y tratamiento de estos pacientes suele ser dificultoso, con múltiples transfusiones y episodios de hospitalización (23). La explicación a este hecho puede ser porque la mayor parte de las lesiones responsables de la HDOO se localizan en intestino delgado (23), zona no accesible a la terapéutica endoscópica convencional la cual ha demostrado que disminuye la tasa de hemorragia persistente, pero además suelen ser pacientes de edad avanzada, muchos de ellos en tratamiento anticoagulante lo que puede explicar los ingresos por hemorragias de repetición $(21,22)$.

Así pues en la actualidad, frente a series de hace 10-15 años (24), el perfil del paciente que ingresa en una Unidad de Sangrantes obedece más al de un paciente con patología de base y polimedicado (anticoagulado, AINE, etc.) o bien al paciente cirrótico con hemorragia por HPT, con un claro descenso del paciente joven ulceroso duodenal. Esperemos que en un futuro próximo, la enteroscopia de doble balón contribuya a filiar la etiología y sobre todo, disminuir la incidencia de hemorragias de repetición procedentes de intestino delgado mediante tratamiento adecuado (25).

\section{BIBLIOGRAFÍA}

1. Laine L, Peterson WL. Bleeding peptic ulcer. N Engl J Med 1994; 331: 717-27.

2. Gilbert DA. Epidemiology of upper gastrointestinal bleeding. Gastrointest Endosc 1990; 36: 8-13.

3. Koelz HR, Arn M. New epidemiology of acute gastrointestinal hemorrhage. Chirurg 2006; 77: 103-10.

4. Dursun M, Yilmaz S, Yukselen V, Canoruc F, Tuzeu A. Analysis of 1242 cases with upper gastrointestinal system bleeding in Sotheastern Turkey: a different etiologic spectrum. Hepatogastroenterology 2005; 52: $1456-8$

5. Schemmer P, Decker F, Dei-Anane G, Hunschel V, Buhl K, Herfarth $\mathrm{C}$, et al. The vital threat of an upper gastrointestinal bleeding: Risk factors analysis of 121 consecutive patients. World J Gastroenterol
2006; 12: 3597-601.

6. Longstreth GF. Epidemiology of hospitalisation for acute upper gastrointestinal hemorrhage: a population-based study. Am J Gastroenterol 1995; 90: 206-10.

7. Kurata JH, Corboy ED. Current peptic ulcer time trends: an epidemiological profile. J Clin Gastroenterol 1988; 10: 259-68.

8. Esrailian E, Gralnek IM. Nonvariceal upper gastrointestinal bleeding: epidemiology and diagnosis. Gastroenterol Clin N Am 2005; 34: 589605.

9. Boonpongmanee S, Fleischer DE, Pezzullo JC. The frequency of peptic ulcer as a cause of upper gastrointestinal bleeding is exaggerated. Gastrointest Endosc 2004; 59: 788-94.

10. Garrido Serrano A, Guerrero Igea FJ, Perianes Hernández C, Arenas Posadas J, Palomo Gil S. Inyección local terapéutica en la úlcera gastroduodenal sangrante: comparación de adrenalina frente a adrenalina más agente esclerosante. Rev Esp Enferm Dig 2002; 94: 401-5.

11. Mahachai V, Thomson AB, Vilaichone RK. Effect of Helicobacter pylori infection and NSADs on the risk of peptic ulcer bleeding. $\mathrm{J}$ Med Asoc Thai 2004; 87: 295-9.

12. Ohmann C, Imhof M, Ruppert C, Jansik V, Vogt C, Fireling T, et al. Time-trends in the epidemiology of peptic ulcer bleeding. Scand J Gastroenterol 2005; 40: 914-20.

13. Graham D, Smith J. The course of patients after variceal hemorrhage. Gastroenterology 1981; 80: 800-6.

14. D’Amico G, de Franchis R. Upper digestive bleeding in cirrosis. Post-therapeutic outcome and prognostic indicators. Hepatology 2003; 38: 599-612.

15. Carbonell N, Pauwels A, Serfaty L, Fourdan O, Levy VG, Poupon R. Improved survival after variceal bleeding in patients with cirrhosis over the past two decades. Hepatology 2004; 40: 652-9.

16. Benvegnu L, Gios M, Boccato S, Alberti A. Natural history of compensated viral cirrhosis: a prospective study on the incidence and hierarchy of major complications. Gut 2004; 53: 744-9.

17. Di Fiore F, Lecleire S, Merle V, Herve S, Duhamel C, Dupan JL, et al. Changes in characteristics and outcome of acute upper gastrointestinal haemorrhage: a comparison of epidemiology and practices between 1996 and 2000 in a multicentre French study. Eur J Gastroenterol Hepatol 2005; 6: 641-7.

18. Morras M, Mondon S, Ortega L, Gual A. Alcoholismo en el hospital general: mortalidad y hospitalizaciones a los 4 años de su detección. Med Clin (Barcelona) 2005; 125: 441-7.

19. The World Health Report 2002. Reducing risks, promoting healthy life. Geneve: World Health Organization; 2003.

20. El-Serag HB, Mason AC. Rising incidence of hepatocellular carcinoma in the United States. N Engl J Med 1999; 340: 745-50.

21. Van Gossum A. Obscure digestive bleeding. Best Pract Res Clin Gastroenterol 2001; 15: 155-74.

22. Grunder S, Straumann A, Pirovino M. Gastrointestinal hemorrhage of unknown origin. Schw Med Woch 1998; 128: 846-9.

23. Zuckerman GR, Prakash C, Askin MP, Lewis BS. AGA technical review on the evaluation and management of occult and obscure gastrointestinal bleeding. Gastroenterology 2000; 118: 201-21.

24. Miño G, Jaramillo JL, Gálvez C, Carmona C, Reyes A, De la Mata M. Análisis de una serie general prospectiva de 3270 hemorragias digestivas altas. Rev Esp Enferm Dig 1992; 82: 7-15.

25. Pérez-Cuadrado E, Mas P, Hallal H, Shanabo J, Muñoz E, Ortega I, et al. Enteroscopia de doble balón: estudio descriptivo de 50 exploraciones. Rev Esp Enferm Dig 2006; 98: 73-81. 